\title{
¿Existe en la universidad una profesionalización docente?
}

\section{Is there a professionalization of teachers in the university?}

\author{
Francisco Imbernón Muñoz \\ Universidad de Barcelona. Barcelona (España) \\ fimbernon@ub.edu \\ Catalina Guerrero Romera \\ Universidad de Murcia. Murcia (España) \\ cgromera@um.es
}

\begin{abstract}
Resumen
Este trabajo presenta una reflexión sobre la profesionalización docente universitaria, la formación y sus implicaciones políticas y educativas, teniendo en cuenta el papel del profesorado en la universidad, en el nuevo contexto social y cultural actual. Se hace referencia a algunos aspectos relacionados con la profesionalización docente universitaria, el ser docente universitario en el siglo XXI y la formación para la innovación docente. A este respecto se indican y cuestionan algunas ideas usuales en la docencia universitaria y se proponen nuevas visiones sobre la formación y la profesionalización docente. El artículo finaliza con una reflexión y sugerencias sobre la formación, la profesionalización y el proceso de innovación docente en la Universidad.
\end{abstract}

\section{Palabras clave}

Profesionalización, formación docente universitaria, innovación docente y Universidad.

\begin{abstract}
This work presents a reflection on the professionalization educational university, the training and his political implications, taking into account the paper of the teacher in the university, in the new social context and cultural current. In this sense, does reference to some appearances related with the professionalization educational university, the be educational university in the 21 st century and the training for the educational innovation. To this respect indicate and question some usual ideas in the university teaching and propose new visions on the training and the professionalization educational. The article finalizes with a reflection and suggestions on the training, the professionalization and the process of educational innovation in the University.

Key words

Professionalization, university educational training, educational innovation and University.
\end{abstract}

\section{Introducción}

La reflexión sobre la profesionalización docente universitaria exige el análisis de muchas variables pero sobre todo del papel del profesorado en la universidad, en el contexto social y cultural actual; en las nuevas competencias científicas, sociales y pedagógicas que se requieren en la profesión de enseñar en esta sociedad llamada "postindustrial" o "del conocimiento", "líquida", "postlíquida" o de las diversas 
denominaciones que se le dan (Bauman, 1999). También requiere un análisis de la formación que se posee para enseñar, y el mejoramiento de las condiciones laborales en la universidad, donde los recortes nos han sumido en un mayor número de profesores de dedicación parcial e inestables en su trabajo y, también, del clima que se está dando en las facultades y departamentos, debido a esos recortes y a la crisis que les acompañó.

En cualquier caso, no cabe duda y, así ha sido destacado por los distintos organismos internacionales, la importancia que tiene la formación del profesorado para la mejora de la calidad de la enseñanza superior y el desarrollo profesional de los y las docentes. Existe una correlación en formación y mejora de los resultados de aprendizaje (Morales, 2013).

La Universidad ha ido cambiado con el tiempo, más de lo que a veces se piensa, y ese proceso de cambio no ha sido rápido, ni fácil, sino complejo y lento. Ya no se trata de enseñar a una minoría homogénea, propia de una época en la cual el conocimiento y su gestión estaban en poder de pocas manos, y monopolizaban el saber, sino a una gran diversidad de estudiantes. Hoy en día los estudios universitarios se han generalizado y su diversidad es un signo de riqueza pero también obliga a un tratamiento diferente de su función social. También se ha perdido el monopolio del saber. El saber ya no está únicamente en la Academia sino en las redes y otras herramientas digitales. Como consecuencia, si la formación de los seres humanos se ha hecho más compleja en los últimos tiempos, la profesión docente universitaria y los procesos de adquisición de conocimientos también se han vuelto más complejos. Esta complejidad se ve incrementada además por el cambio radical y vertiginoso de las estructuras científicas, sociales y educativas (Barnett, 2001 y 2002).

Por tanto, los vertiginosos cambios que se han producido en las últimas décadas han sido muy importantes, tanto en el ámbito psicopedagógico (nueva concepción del trabajo, el papel de la Universidad en una escolarización democrática, la aparición y extensión de nuevas tendencias científicas, los nuevos conceptos sobre el aprendizaje, la aparición de las competencias,...), como en el social (la escolarización generalizada, el acceso cultural masivo, el apogeo de las tecnologías de la información y la comunicación, el cambio y la crisis institucional de instancias históricas de socialización, la crisis de legitimación de la modernidad,...). Así como se ha ido generalizando el aprendizaje a lo largo de toda la vida (lifelong learning) y la instauración relativa del Espacio Europeo de Educación Superior con sus fortalezas y debilidades (Comisión Europea, 2003). Al fin y al cabo son elementos de análisis importantes en la generación de nuevas alternativas de futuro en la profesionalización docente universitaria. No podemos generar una profesionalización docente a partir de modelos de enseñanza obsoletos que funcionaron (aunque fuera con problemas) en otros tiempos. No hay soluciones viejas y anticuadas para problemas nuevos.

Y queremos reiterar el importante cambio del alumnado que llega a las aulas universitarias. Las repercusiones de los nuevos sistemas educativos y sociales configuran un nuevo tipo de alumnado. El hecho de no partir de la premisa de una formación diferente para un alumnado diferente, sería un grave error en los planteamientos de la docencia universitaria. Se habla de la universidad centrada en el aprendizaje del alumnado. Pero ¿qué significa? Poner en crisis la formación teoricista

¿Existe en la universidad una profesionalización docente? Francisco Imbernón Muñoz y Catalina Guerrero Romera.

Página 2 de 12 
centrada en el docente. En numerosas ocasiones la dinámica del aula universitaria se basa únicamente en la lección magistral, en la sesión puramente expositiva del docente, la palabra (y hoy en día en las presentaciones matando, a veces, al alumnado de Power Point o similar) y los apuntes son la base del estudio. Se necesita un cambio en la actitud y la metodología docente. Ya no sólo son numerosas las voces que apelan por un cambio en la metodología docente, sino que también cada vez encontramos nuevas experiencias para paliar esta situación, que suele ser motivo de discusiones en los diversos foros universitarios (aprendizaje autónomo, metodologías activas, participación, colaboración, autoevaluación, autorregulación, aprendizaje virtual, aprendizaje basado en problemas, aula inversa, gamificación, etc.).

\section{Profesionalización Docente}

Desde hace años, se manifiesta un interés creciente por la profesión docente universitaria y su formación, como se comprueba en los servicios de formación e innovación aparecidos en todas las universidades y la aparición de publicaciones sobre cuestiones relativas a la enseñanza universitaria, el profesorado y el papel de la innovación y los proyectos de innovación docente.

Y eso es bueno, aunque somos conscientes que aún la preocupación por la docencia en la universidad es menor que por la investigación (también debido a causas contextuales de promoción universitaria), pero algo está cambiando. Es necesario continuar haciendo una reflexión y un análisis sobre aspectos globales y específicos de la profesión docente universitaria que nos permitan tener una visión de conjunto de la temática. Una reflexión global que estuviera por encima de los aspectos más técnicos y científicos de la profesión y que, a la vez, una reflexión más específica que nos diera elementos de mejora para introducir en las políticas y las prácticas de la profesionalización del profesorado universitario y su formación. Este análisis podría pasar por:

- Análisis del concepto de profesión docente universitaria que predomina en la sociedad actual y en la mentalidad del profesorado: qué principios son derivados del entorno socioeconómico, de las creencias y valores y de las disciplinas científicas, así como del modelo profesional y de formación y de las funciones específicas del profesorado.

- Análisis de los cambios sociales para entrever, dentro de lo posible, cuál será la función de la universidad y de las competencias del profesorado.

- Análisis de la situación actual de la universidad para ver si cumple las funciones por la que fue creada o sus estructuras y procesos institucionales (organización, funcionamiento, participación, etc.) son obsoletas en la sociedad de hoy en día.

- Análisis de la situación actual de las políticas, de los planes de estudio y de la organización con objeto de deducir las estrategias adecuadas en las competencias curriculares necesarias del profesorado.

¿Existe en la universidad una profesionalización docente? Francisco Imbernón Muñoz y Catalina Guerrero Romera.

Página 3 de 12 
- Análisis del alumnado actual que permita revisar las funciones profesionales del profesorado y las nuevas tareas que habrá que asumir dentro y fuera de la universidad.

- Análisis de la situación laboral y la carrera docente del profesorado desde el punto de vista individual y colectivo, para ver cuáles son las mejoras que habrá que introducir para ejercer la profesión (acceso, relaciones laborales, retribución, etc.).

Todo esto nos permitiría concretar funciones, competencias y procesos de profesionalización para dar respuesta, no de forma tradicional y reproductora, sino de forma alternativa y de prospectiva de futuro a la práctica profesional y a las necesidades del profesorado universitario. Aunque este análisis total es imposible en el presente artículo, intentaremos, centrándonos en el profesorado, dar algunos elementos que lo faciliten.

\section{Ser docente universitario en el siglo XXI}

Damos por hecho que las universidades han evolucionado a lo largo del siglo XX, pero lo han hecho sin romper las líneas directrices que se le marcaron en su nacimiento: transmisora cultural, exclusiva, seleccionadora, cultura individualista del profesorado, etc. La universidad ya no es «un lugar» exclusivo puesto que, cada vez más (y esta es una de las grandes diferencias con siglos anteriores), comparte el poder de la transmisión del conocimiento con otras instancias socializadoras: televisión, Internet, medios de comunicación de todo tipo, redes, cultura social más grande, educación no formal, etc., y la universidad necesita una revisión desde el momento que ha perdido el monopolio del saber (Marcovitch, 2002; Michavila, 2001).

Como decíamos anteriormente, si la formación de los seres humanos es más compleja, la profesión docente también lo será. Y además, esta complejidad se ve incrementada por el cambio radical y vertiginoso de las estructuras científicas y sociales.

El profesorado universitario de principios del siglo XXI no tiene nada que ver con el de principios del siglo XX, por lo tanto, la universidad y la profesión docente se desarrollan en un contexto que ha variado sustancialmente y que influirá en la profesión:

- Un incremento acelerado y un cambio vertiginoso en las formas que adopta el conocimiento científico y en los productos del pensamiento, la cultura y arte.

- Una evolución acelerada de la sociedad en sus estructuras materiales, institucionales y formas de organización de la convivencia, modelos de familia, alternativas políticas, modelos de producción y de distribución, que se reflejan en el cambio inevitable de las actuales maneras de pensar, sentir y actuar de las nuevas generaciones. Elementos nuevos, como por ejemplo la aparición de la nueva economía, la tecnología que

¿Existe en la universidad una profesionalización docente? Francisco Imbernón Muñoz y Catalina Guerrero Romera.

Página 4 de 12 
desembarca con gran fuerza, la globalización se hace patente, etc. Empieza a surgir una gran crisis de la profesión docente.

- Unos contextos sociales que condicionarán la universidad y reflejarán una serie de fuerzas en conflicto. Los vertiginosos cambios de los medios de comunicación y tecnológicos han ido acompañados de profundas transformaciones en la vida personal e institucional de muchas organizaciones y personas, y han puesto en crisis la transmisión del conocimiento y, por lo tanto, también la universidad. Un análisis de la enseñanza que ya no es considera patrimonio exclusivo de los docentes, sino de más profesionales $\mathrm{y}$ de los medios de que la sociedad, estableciendo nuevos modelos relacionales y participativos en la práctica de la enseñanza mediante redes de conocimiento.

- Los procesos, la organización y los medios de enseñanza no funcionan del todo, los edificios universitarios no son adecuados para una nueva manera de ver la enseñanza universitaria actual (García y Mondaza, 2002).

Y, además, durante las últimas décadas, hemos visto como se ha ido cuestionando el conocimiento inmutable de las ciencias y se abren otras concepciones en las cuales la interpretación, la comprensión de la realidad, la incertidumbre y la complejidad (Barnett, 2002) tienen un papel importante en la realidad científica y social. También se han ido incorporando nuevos discursos relacionados con los aspectos éticos, relacionales, colegiales, actitudinales, emocionales, el uso reflexivo de la tecnología, etc., todos los cuales son necesarios para conseguir una mejor enseñanza universitaria (Hannan \&Silver, 2002).

También conviene estar alerta para impedir una fagocitación de todos estos elementos por parte de los sistemas económicos y de la regulación del mercado, para introducir elementos que puedan suponer procesos universitarios de autonomía vigilada, de profesorado dependientes y de valores insolidarios o fugaces.

Cada vez más, necesitaremos una renovada universidad y una nueva forma de enseñanza en unas nuevas estructuras didácticas y organizativas, y esto requiere una reconceptualización importante de la profesión docente universitaria, y, también, una asunción de nuevas competencias profesionales. Y esto comporta una nueva manera de ejercer la profesión y de formar de forma diferente al profesorado universitario en esta compleja sociedad del futuro (Bain, 2007). Es decir, los nuevos tiempos requieren una enseñanza y un profesional muy diferente, puesto que la profesión docente asume nuevas funciones. Este profesional docente universitario tendrá que ser competente para realizar diversidad de tareas:

- Mediador en el proceso de enseñanza-aprendizaje individual y de grupos.

- Conocedor disciplinario además de planificador y proyectista curricular, teniendo en cuenta la diversidad de componentes que intervienen en el proceso

¿Existe en la universidad una profesionalización docente? Francisco Imbernón Muñoz y Catalina Guerrero Romera.

Página 5 de 12 
de enseñanza, la realidad institucional y los obstáculos que los acondicionamientos contextuales comportan.

- Tener una actitud colegial para trabajar con un equipo de personas (equipos docentes) y en un contexto específico (ecosistema cultural). El proceso de aprendizaje basado en el alumnado, al ser más complejo que en el pasado, tiene que superar la fase en la cual se consideraba una simple transmisión de conocimientos.

Todo esto nos trae a una necesidad, por parte del profesorado, de un conocimiento más profundizado de sí mismo (valores, creencias, supuestos...), del entorno a su trabajo universitario (social, político, cultural...), del conocimiento y las destrezas que tiene que transmitir, de los constructos teóricos de la enseñanza-aprendizaje y del currículum (planificación, metodología, organización, materiales,...).

\section{4. ¿Y la formación para la innovación docente?}

El cambio en el profesorado universitario, al ser un cambio en la cultura profesional, es muy lento. Pero esta lentitud comporta también una necesidad de vivir personalmente la experiencia de cambio, ya sea individual y colectivamente. Los cambios de los otros no necesariamente ayudan al cambio de uno pero ver el cambio en otros puede provocar esa importante reflexión sobre lo qué se hace. Para cambiar la universidad se ha de formar al profesorado, pero no es suficiente, el cambio siempre va acompañado del cambio del contexto, de dónde se trabaja y se realiza la acción práctica. Si se desea cambiar la universidad se ha de cambiar al profesorado y el contexto donde trabaja. Es una acción colectiva.

Provoca más innovación unir la formación a la realidad del contexto. La formación para la innovación tiene que tener en cuenta que, más que actualizar una persona y enseñarle, tiene que crear las condiciones para que esta aprenda. La formación por sí misma hace poco si no está unida a cambios del contexto, organizativos, de gestión y de relaciones de poder. El desarrollo profesional del profesorado universitario no recae en la formación, sino en varios componentes que se dan conjuntamente en la práctica laboral.

Es posible que haya más formación y poco cambio. Aunque se ha ido aumentando la formación aún predomina la formación transmisora y estándar, con una supremacía de una teoría a veces descontextualizada, alejada de los problemas prácticos del profesorado que asiste a la formación, basada en un profesorado universitario medio o generalista que no existe. Y también, a pesar del discurso en las políticas universitarias que la formación en docencia e innovación es necesaria, en la práctica las universidades (y a veces la cultura docente del profesorado) le dan una importancia relativa.

La profesionalización docente en la universidad tendría que apoyarse en una reflexión de los sujetos, de forma individual y colectiva, sobre su práctica docente, de forma que les permita examinar sus teorías implícitas, sus esquemas de funcionamiento, sus

¿Existe en la universidad una profesionalización docente? Francisco Imbernón Muñoz y Catalina Guerrero Romera.

Página 6 de 12 
actitudes,..., realizando un proceso constante de autoevaluación que oriente el desarrollo profesional (Escudero, 1999).

No se puede ver al profesorado universitario actual como únicamente un transmisor científico, sino un profesional que transmite conocimientos válidos, útiles y pertinentes pero también un profesional que tiene capacidades de procesamiento de la información, análisis y reflexión crítica, decisión racional, evaluación de procesos y reformulación de proyectos, tanto laborales como sociales y educativos en su contexto y con sus colegas universitarios (Brockbank \& Mcgill, 2002).

Una formación para la innovación docente requeriría entonces el desarrollo de modelos y entornos de trabajo, aprendizaje y experimentación en sintonía con el contexto y los modelos actuales: educación abierta, transformación y adaptación digital, metodologías y modos de enseñanza-aprendizaje y de construcción y gestión del conocimiento más interactivos, participativos y colaborativos (Osuna, 2014) que conecten con los retos actuales, con las necesidades y problemas de las instituciones universitarias y del alumnado y se avance hacia una innovación sistémica, más abierta y disruptiva (Christensen, Baumann, Ruggles \& Sadtler, 2006).

En este sentido, debemos actualizar e impulsar nuevos modelos de formación del profesorado, más abiertos, activos y que fomenten la creación de espacios y escenarios de aprendizaje, a través de equipos docentes que trabajen juntos colaborativamente y que desarrollen nuevas fórmulas o modalidades para la formación docente: investigacción-acción, intercambio de experiencias, comunidades de aprendizaje,..., (Herrán y Paredes, 2010). Todo ello orientado a contribuir a la mejora de las competencias docentes de profesionales reflexivos y autónomos que aprenden y mejoran investigando colaborativamente sobre su propia práctica profesional (Imbernón, 2012).

En los últimos años hay una preocupación por la innovación docente del profesorado. Las universidades cuentan con multitud de acciones y convocatorias de innovación bajo las cuales se desarrollan proyectos y prácticas de innovación docente en las que se experimentan, analizan y explotan recursos y resultados diversos. Todo ello exige una reflexión y una evaluación más profunda de su utilidad, aplicabilidad y calidad, con la finalidad de valorar su impacto real en la comunidad universitaria y en la sociedad para que sea innovación (Guerrero, 2017). Fidalgo Sein-Echaluce, Lerís, \& García-Peñalvo afirmaban:

Pero nada más lejos de la realidad, las prácticas se suelen repetir, incluso en la propia universidad, y el profesorado, en general, no tiene indicadores válidos para conocer el grado de innovación educativa que realiza. Además, cuando se divulgan las prácticas es muy difícil encontrar datos que permitan aplicarlas en otros contextos, valorar su exportabilidad y aplicabilidad (Fidalgo et al., 2013, p. 751).

Si deseamos una formación para la innovación docente podríamos preguntarnos qué formación para la innovación queremos y qué formación para la innovación necesitamos en el contexto a partir de la evaluación e identificación de buenas prácticas que se estén realizando y de un perfil de competencias profesionales en el campo de la

¿Existe en la universidad una profesionalización docente? Francisco Imbernón Muñoz y Catalina Guerrero Romera.

Página 7 de 12 
innovación docente. Perfil orientado a las destrezas y las habilidades que requerirán los docentes desde una nueva reconceptualización de la profesión docente universitaria, y de una nueva asunción de competencias profesionales en este ámbito.

Se coincide en subrayar que en la era del aprendizaje el reto está en docencia de alta calidad a través de tecnología e innovación con los docentes como activadores clave de los aprendizajes de los estudiantes (Biggs, 2003) y de los procesos de innovación docente y aplicaciones tecnológicas. Francesc Pedró, Director del Centro de Investigación e Innovación Educativas de la OCDE, pone el énfasis en la importancia que tiene el docente y en las pedagogías que permiten o están detrás de los usos y aplicaciones de las innovaciones y los dispositivos tecnológicos para la educación. Subraya asimismo la necesidad de apoyarles para que desarrollen sus procesos de innovación y puedan establecer unos itinerarios de aprendizaje que lleven a los estudiantes a aprender más y mejor a partir de su propio proceso de empoderamiento (Pedró, 2015).

La Formación docente debe de contribuir a generar esa innovación docente de calidad y que tenga un impacto en el aprendizaje de los estudiantes y de las instituciones universitarias. La responsabilidad como docentes es contribuir a despertar inquietudes y curiosidades por el conocimiento y el aprendizaje, combatiendo los prejuicios y superando los diferentes obstáculos que se presentan, algunos descritos aquí, si realmente se quiere una formación para la innovación docente que estimule y refuerce los cambios señalados.

\section{Conclusiones}

Todo lo anterior nos lleva a que la formación pedagógica del y la docente universitaria debería pasar por favorecer en el debate y la construcción de unas bases reales sobre las cuales construir proyectos de innovación docente intentando eliminar al mismo tiempo los procesos de atomización, gremialismo e individualismo en el trabajo profesional muy típico de la cultura académica del docente universitario (y que los cursos estándar pueden continuar promoviendo). También es preciso apoyar la experimentación y la difusión de materiales de grupos de innovación más reducidos y homogéneos, aunque sean de carácter interdepartamental o interfacultativo, en la línea de proporcionar referencias y elementos de dinamización que surgen del profesorado. Otras acciones en este sentido deben estar encaminadas a favorecer la formación de equipos docentes donde sea posible un mayor avance, mediante propuestas concretas en las asignaturas y en los planes docentes.

Parece ser que, en el mundo actual, lo único no mutable es el cambio. La formación no sirve ya únicamente para "estar al día", para actualizarse, sino como un elemento intrínseco en las profesiones para interpretar y comprender ese cambio constante. Como se ha ido comentando, la profesión docente universitaria se mueve, hoy día, en contextos sociales que reflejan una serie de fuerzas en conflicto, divergencias, dilemas, dudas y situaciones contextuales y de incertidumbre. La formación puede ser un elemento revulsivo importante para interpretar y comprender esa incertidumbre $\mathrm{y}$

¿Existe en la universidad una profesionalización docente? Francisco Imbernón Muñoz y Catalina Guerrero Romera.

Página 8 de 12 
entender lo que pasa. Y ayudar a arriesgarse al cambio, a ver más allá de nuestros límites, a superar esa ceguera del conocimiento que indica Morin (2002).

Pero la formación docente universitaria no puede ser obligatoria (al menos en lo referente a la formación permanente), necesita transitar por concienciar al profesorado de la necesidad pedagógica para mejorar la relación con la transmisión de la disciplina (el conocimiento didáctico del contenido académico), consigo mismo y con el alumnado. Ello implica un cambio en los posicionamientos de las estructuras organizativas universitarias $\mathrm{y}$, también, cognitivas del profesorado universitario, asumiendo una mayor implicación individual y colectiva en procesos de reflexión e investigación sobre los efectos de la docencia universitaria para comprender las prácticas docentes y las situaciones en que estas se desarrollan.

Uno de los obstáculos de la extensión institucional de la formación en docencia universitaria es cómo romper inercias e ideologías institucionales obsoletas (la formación siempre está sujeta y vinculada a marcos teóricos y a supuestos ideológicos). Romper con imaginarios, sociales y personales, muy asentados en las estructuras docentes universitarias. Ello requiere, más allá de cursos de formación, una reestructuración, un repensar importante de la Universidad, de la formación en docencia universitaria y de la profesionalización docente universitaria.

Pero se ha de tener en cuenta que la mejora de la docencia universitaria no depende únicamente de la metodología utilizada en las aulas universitarias, sino de la implicación institucional de la Universidad y del colectivo docente. Tratar únicamente los aspectos técnicos de la docencia universitaria, como una cierta pasión en lo metodológico como cura de todos los males académicos, puede llevarnos a una inducción a la obsolescencia, a una reproducción banal o a una visión estereotipada del conocimiento pedagógico.

En esa reestructuración epistemológica de la docencia universitaria será fundamental revisar el funcionamiento de las facultades y departamentos (trabajo organizativo, toma de decisiones, relaciones de poder, comunicación, participación...); buscar alternativas y reflexionar sobre la docencia para no caer en prácticas reproductoras y sobre la evaluación del alumnado y aprender a trabajar colegialmente, elaborar proyectos de (auto)formación e innovación contextualizados.

Muchos de los elementos pedagógicos que intervienen en la docencia son difíciles de enseñar y, por tanto, más que enseñarse deberían aprenderse en la reflexión sobre la práctica docente. Facilitar esos espacios de reflexión, participación y formación es la función imprescindible de la formación en docencia universitaria. Más que asumir una función de actualización pedagógica del profesorado universitario, la formación en docencia universitaria debería asumir esa creación de espacios de aprendizaje. Ayudar a repensar la práctica docente desde la conciencia de la contextualización y la complejidad del acto educativo. $\mathrm{Y}$ eso es lo que puede provocar introducirse en proyectos de innovación docente.

También la formación necesita un poco de imaginación y utopía. La utopía es aquello que pretendemos y desearíamos para un futuro pero que consideramos difícil de

¿Existe en la universidad una profesionalización docente? Francisco Imbernón Muñoz y Catalina Guerrero Romera.

Página 9 de 12 
alcanzar con los medios actuales. Pero no es una imposibilidad, sino que permite establecer los elementos para que en un futuro se pueda conseguir. Aun así, la utopía educativa es patrimonio del profesorado, de la libertad del pensamiento docente, sin utopías no tendríamos el mundo y las circunstancias educativas que hoy día poseemos.

Y todo ello acompañado de elementos de formación y autoformación: intercambio de experiencias, formación contextualizada, publicaciones, formación en línea,... Pero, ello será realmente una utopía si no se sensibiliza y corresponsabiliza a las instituciones académicas y, sobre todo, al profesorado universitario, de la importancia para su profesión de la formación en docencia y en innovación universitaria.

Pero para que la universidad pueda desarrollar a la vez una docencia no devaluada, una investigación e innovación docente útil y una difusión del conocimiento en condiciones, se deberían poner en marcha diversas medidas. Aumentar los recursos económicos y humanos, preocuparse por la relación enseñanza-aprendizaje y evaluación, modificar las relaciones de autoridad, analizar el saber y poder en los departamentos, crear la posibilidad de formarse y autoformarse en la impartición docente de la disciplina, especializarse en la oferta formativa, etc. Es imprescindible romper con la homogeneización de nuestra universidad; es necesaria la aparición de proyectos nuevos. La universidad no debe reproducirse a sí misma, sino que debe diversificarse para abarcar más necesidades y nuevos campos científicos y también, ofrecer nuevos servicios públicos.

Por tanto, deberíamos ser más conscientes del compromiso social y de la necesidad de revisar los procesos formativos y de romper el modelo de aula cerrada, de repetición académica, de redundancia extenuante, ya que todo ello no sólo genera una labor individual, sino que ocasiona ciertos problemas de comunicación entre el profesorado y también con el alumnado, e incluso impedir que tenga lugar un fenómeno imprescindible en toda labor profesional como es el intercambio colaborativo de la propia teoría práctica docente con los que te rodean.

Y, por supuesto, sensibilizar y corresponsabilizar al profesorado en la importancia de esa formación. Si no existe preocupación de mejora, actitudes positivas institucionales y políticas respecto a la formación, redes de relación para el conocimiento e intercambio de innovaciones didácticas y propuestas alternativas, la formación servirá para muy poco o únicamente para la inhibición o para acreditar algún mérito académico.

Presentación del artículo: 23 de octubre de 2017

Fecha de aprobación: 18 de enero de 2017

Fecha de publicación: 31 de enero de 2017

Imbernón Muñoz, F., y Guerrero Romera, C. (2017). ¿Existe en la universidad una profesionalización docente? RED. Revista de Educación a Distancia, 56.

Consultado el (dd/mm/aaaa) en

http://www.um.es/ead/red/56/imbernon_guerrero.pdf

¿Existe en la universidad una profesionalización docente? Francisco Imbernón Muñoz y Catalina Guerrero Romera.

Página 10 de 12 


\section{Financiación}

Esta investigación no ha recibido ninguna subvención específica de los organismos de financiación en los sectores públicos, comerciales o sin fines de lucro.

\section{Referencias bibliográficas}

Barnett, R. (2001). Los límites de la competencia. La educación superior y la sociedad. Barcelona: Gedisa.

Barnett, R. (2002). Claves para entender la Universidad en una era de supercomplejidad. Girona: Pomares.

Bain, K. (2007). Lo que hacen lo mejores profesores universitarios. Valencia: Publicaciones de la Universidad de Valencia, $2^{a}$ ed.

Bauman, Z. (1999). Modernidad líquida. Buenos Aires: Fondo de Cultura Económica.

Biggs, J. (2003). Calidad del aprendizaje universitario. Cómo aprenden los estudiantes. Madrid: Narcea.

Brockbank, A y McGill, I. (2002). Aprendizaje reflexivo en la educación superior. Madrid: Morata.

Comisión Europea (2003). El papel de las universidades en la Europa del Conocimiento. Bruselas: Comisión de las Comunidades Europeas.

Christensen, C. M., Baumann, H., Ruggles, R., \& Sadtler, T. M. (2006). Disruptive innovation for social change. Harvard Business, Review, 84 (12), pp. 94-101.

Escudero, J. M. (1999). La formación permanente del profesorado universitario: cultura, política y procesos. Revista Interuniversitaria de Formación del Profesorado, 34(abril), 133-157.

Fidalgo, A.; Sein-Echaluce, M.L.; Lerís, D.; \& García-Peñalvo, F.J. (2013). Sistema de Gestión de Conocimiento para la aplicación de experiencias de innovación educativa en la formación. Madrid. II Congreso Internacional sobre Aprendizaje, Innovación y Competitividad (CINAIC 2013), pp. 750-755.

García, J. y Monzaza, G. (2002). Jóvenes, universidad y compromiso social. Madrid: Narcea.

Guerrero, C. (2017). Evaluación de las prácticas y proyectos de innovación docente en el contexto universitario. Modelo e+iDu. XIV Foro Internacional sobre la Evaluación de la Calidad de la Investigación y de la Educación Superior (FECIES). Granada, 22-24 de Junio.

Hannan, A., \& Silver, H. (2003). Innovar en la Universidad. Madrid: Narcea.

Herrán, A. y Paredes, J (2010). Desarrollo profesional de los docentes, innovación y mejora de la enseñanza universitaria. En Herrán, A.; Pérez, F. y Díaz, V. (coords.). La formación del profesorado de la Universidad Alfonso X el Sabio ante el reto de Bolonia. Madrid: Fundación UAX, pp. 203.235.

¿Existe en la universidad una profesionalización docente? Francisco Imbernón Muñoz y Catalina Guerrero Romera.

Página 11 de 12 
Imbernón, F. (2012). ¿Hay nuevas tendencias en la formación del profesorado? Un congreso para debatirlo. Aula de Innovación Educativa, no 212, pp. 14-17.

Marcovitch, J. (2002). La universidad (im)posible. Madrid: Cambridge Press y OEI.

Michavila, F. (2001). La salida del laberinto. Crítica urgente de la Universidad. Madrid: Editorial Complutense.

Morales, A.; Edith, L; Hidalgo, C.; García, R. Molinar, J. (2013). Relación entre estilos de aprendizaje, rendimiento académico y otras variables relevantes de estudiantes universitarios. Revista Estilos de Aprendizaje, nº12, Vol. 11, pp.1-16.

Morin, E. (2002). Los 7 Saberes Necesarios para la Educación del Futuro. Barcelona: Paidós.

Osuna, S. (Coord.) (2014). Escenarios virtuales educomunicativos. Barcelona: Icaria.

Pedró F. (2015). Los profesores de hoy en día les dan mil vueltas a los que había antes. Recuperado de http://www.elconfidencial.com/alma-corazon-vida/2015-0223/francesc-pedro-los-profesores-de-hoy-en-dia-les-dan-mil-vueltas-a-los-quehabia

¿Existe en la universidad una profesionalización docente? Francisco Imbernón Muñoz y Catalina Guerrero Romera. 